\title{
A Multi-agent Approach to Providing Different Forms of Assessment in a Collaborative Learning Environment
}

\author{
Mitra Mirzarezaee $^{1,3}$, Kambiz Badie $^{1}$, Mehdi Dehghan $^{2}$, and Mahmood Kharrat ${ }^{1}$ \\ 1 Iran Telecommunication Research Center (ITRC) \\ \{k_badie, kharrat\}@itrc.ac.ir \\ 2 Dept. of Computer Eng., Amirkabir University of Technology \\ dehghan@ce.aut.ac.ir \\ 3 Dept. of Computer Eng., Islamic Azad University-Science and Research Branch \\ m_rezaee@itrc.ac.ir
}

\begin{abstract}
This paper proposes a multi-agent framework that facilitates provision of different forms of assessment by means of an integrated basis for comparative analysis of different forms of assessment. It is adaptive in the sense that it automatically changes forms of assessment to reach better performance and learning outcomes. The proposed system can be tuned to different contexts and learning materials.
\end{abstract}

\section{Introduction}

A collaborative learning environment is an environment that allows participants to collaborate and share access to information, instrumentation, and colleagues [1]. It is recognized that the main goal of professional education is to help students develop into reflective practitioners who are able to reflect critically upon their own professional practice. Assessment is now represented as a tool for learning, and present approaches to it focus at one new dimension of assessment innovation, namely the changing place and function of assessor. Therefore alternatives in assessment have received many attentions in the last decade, and with respect to this, several forms of more authentic assessments such as skills of self-, peer- and co-assessment are introduced [4].

As building assessment systems in different contexts and for different forms of assessment is a very expensive, exhaustive and time-consuming process [2,3], a multi-agent approach to design an Intelligent Assessment System, has been used that provides three advantages for the developers: easier programming and expansion, harmless modification, and distribution of the system within different computers [2].

In the next sections, the proposed multi-agent framework and its components are introduced, and finally arguments of possibility and applicability of the system are presented.

\section{The Proposed Multi-agent Framework}

The proposed framework, which enables the construction of different forms of assessment within a single integrated skeleton, is a two-layered architecture and its general 
schema is illustrated in Figure 1.The first layer is called the test layer, which is similar to the general multi-agent architecture of an Intelligent Tutoring System, but also concerns the basic requirements of an assessment process. The second layer is called the assessor layer, and is responsible to set the best form of assessment for the current situation based on the decision made by the test administrator or the critic agent.

\subsection{Test Layer}

This is the main underlying part of the system, where selected theory of measurement, methods of adaptive testing, activity selection, response processing and scoring exists. The task library is a database of task materials (or references to such materials) along with all the information necessary to select, present, and score the task. The test layer consists of four different agents (tutor, assessor, student model and presentation), each of which has its own responsibilities.

Tutor agent is responsible for managing and administrating the tests. Estimation of item parameters, test calibration, equation and selection of the next task to be represented to the user, are among its main responsibilities.

Assessor agent is responsible for response processing (key matching) and also estimation of students' abilities according to their obtained raw scores. This agent focuses on aspects of the student response and assigns them to categories. The results of assessor agent estimations of learners' abilities are used as the criterion for evaluation of results obtained from other forms of assessment.

Student Model agent is responsible for modeling individual students' knowledge and abilities in that special domain.

Presentation agent is responsible for presenting the task to the examinee and also collecting his/her responses.

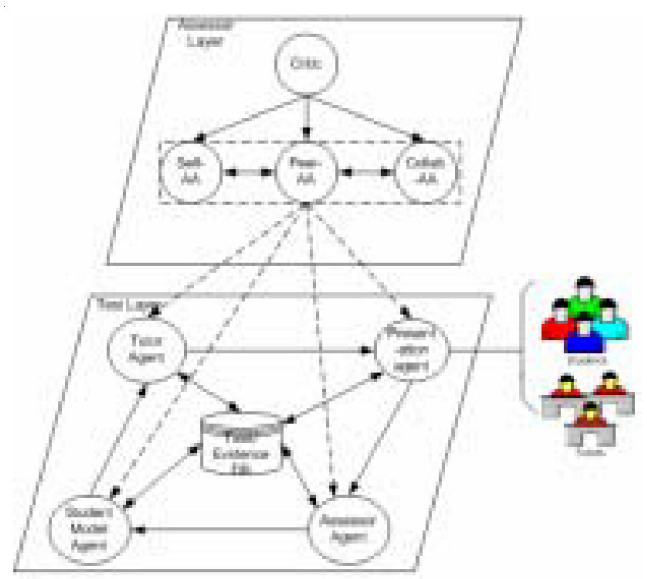

Fig. 1. A multi-agent architecture for implementing different forms of assessment 


\subsection{Assessor Layer}

The assessor layer, comprising of three different assessor agents and one critic, has the duty of identifying and setting the best form of assessment. The minimum required assessor agents are self-, peer- and collaborative assessors. This layer has the ability to perform each of the nine mentioned forms of assessment by activating one or more of the agents simultaneously.

The critic agent as its name says, is responsible for deciding on the best possible forms of assessment or a combination of them according to the involving factors.

\section{Concluding Remarks}

The framework envisioned in this paper is an environment where non-co-located learners can gather and interact with each other to reach goals of assessments. One can construct a class of students from different parts of the world, whom can be assessed according to the modern learner-centered methods of assessment and can benefit from the advances of technology to attend more reliable learning courses and receiving feedbacks of their peers, and tutors. Also, they can evaluate themselves and finally reach a better agreement on their abilities and failures.

The proposed framework has certainly some other advantages: First, it can be seen as a general standard framework of assessment that can be easily added to the former designs with fewer modifications. Secondly, educational researches can benefit from having an integrated basis for comparative analysis of different forms of assessment, which, not only brings them more accuracy and precisions in research outcomes, but also reduces the complexity of their work. And finally, using artificial intelligence techniques, it can be the basis for building an adaptive assessment system that changes its forms of assessment to reach better performance and learning outcomes accordingly.

To sum up, for maintaining different forms of learner assessment, where a variety of possible forms of assessment exists, uniformity is needed from which we can converge in several directions. With this purpose in mind, we proposed an integrated multi-agent framework that enables provision of different forms of assessment. In designing the proposed system, we considered to be consistent with general multi-agent frameworks of Intelligent Tutoring Systems.

\section{References}

1. M.c. Dorneich, P.M. Jones, The Design and Implementation of learning collaboratively, IEEE International Conference on Systems Man and Cybernetics, (2000).

2. M. Badjonski, M. Ivanovic, Z. Budimac, Intelligent Tutoring System as Multi-agent System, IEEE International Conference on Intelligent Processing Systems,(1997).

3. L.M.M. Giraffa, R.M. Viccari, The use of Agents Techniques on Intelligent Tutoring Systems, IEEE International Conference on Computer Science,SCCC'98, (1998).

4. D. Sluijsman, F. Docky, G. Moerkerky, The use of self-,peer- and co-assessment in higher education a review of litreture, Studies in Higher Education, Vol. 24, No. 3, (1991), p. 331. 